\title{
Guest editorial: Special Issue on Biomedical Tribology
}

\author{
Guoxin XIE, Jianbin LUO \\ State Key Laboratory of Tribology, Tsinghua University, Beijing 100084, China \\ (C) The author(s) 2021.
}

Biomedical tribology has developed rapidly with the joint efforts of medical and tribological researchers, and it concerns various kinds of friction that existed inside and outside the body, such as the friction of joints, the friction between skin and clothing, and the friction in the lumen (blood vessels, trachea, digestive tube, and excretory tube), which causes many physiological changes and diseases in the human body. Besides, many medical devices have been used to treat or alleviate various diseases. Biomedical tribology plays a vital role in solving the widespread tribological issues in the medical devices field, aiming to improve the performance of medical devices and prolong the service life.

This special issue of Friction aims to introduce readers to the field of biomedical tribology, not only covering the basic understanding of biomedical tribology but also its applications in medical devices. To demonstrate the breadth and timeliness of the topic and provide opportunities for the publication of new discoveries, 12 papers by tribologists and scientists were selected, including one review article on the bio-tribology of medical devices, one research papers on the corrosionwear behavior of biocompatible magnesium matrix composite, one research paper on the electrostatic attraction at gecko setae, two research papers on the preparation of hydrogels with specific bio-medical purposes, three research papers on the lubrication of joints, one research paper on the microvascular hemostasis, three research papers on the friction behaviors of coatings and materials applied in vivo or in vitro.

The first paper by Zhang et al. reviews the biotribological issues involved in commonly medical devices, and summarizes the current understanding of the bio-tribological behavior and mechanism in medical devices.

As a new generation of biocompatible implant materials, magnesium $(\mathrm{Mg})$ alloys presents excellent biocompatibility and relatively low elastic modulus. However, the excessive degradation rate and poor wear resistance in the human body limit its biomedical application. The original research paper "Corrosionwear behavior of a biocompatible magnesium matrix composite in simulated body fluid" chooses hydroxyapatite $\left(\mathrm{Ca}_{10}\left(\mathrm{PO}_{4}\right)_{6}(\mathrm{OH})_{2}\right.$, $\left.\mathrm{HA}\right)$ to improve corrosion-wear behavior of $\mathrm{Mg}$ alloy (ZK60).

The paper "Electrostatic attraction caused by triboelectrification in climbing geckos" analyzes the electrostatic attraction at gecko setae. The results indicate the adhesion driven by triboelectrification only accounts for $\sim 1 \%$ (experiments) and $\sim 3 \%$ (model) of total adhesion. Their study deepens the understanding of gecko adhesion mechanism and contributes to the development of fibular adhesive.

Hydrogel is a kind of promising soft and wet material, which has a wide range of applications in the fields of tissue engineering and biomedicine. However, due to the poor mechanical properties, traditional hydrogels exhibit poor tribological performance under high loads. In the paper titled "Fibers reinforced composite hydrogels with improved lubrication and load-bearing capacity", the authors combine three kinds of fibers with Poly(n-vinyl pyrrolidone)poly(2-hydroxyethyl methacrylate) (PVP-PHEMA) hydrogels to improve its mechanical and tribological properties. Among three reinforced hydrogels, the needle-punched fiber hydrogel has the highest loadcarrying capacity and low friction performance, and is expected to become a new biomimetic lubricating material. The paper "Robust and conductive hydrogel based on mussel adhesive chemistry for remote

* Guest Editors: Guoxin XIE, E-mail: xgx2014@tsinghua.edu.cn; Jianbin LUO, E-mail: luojb@tsinghua.edu.cn 
monitoring of body signals" uses potato residues and chitosan as scaffold materials to prepare a hydrogel with excellent mechanical properties, as well as a remarkable self-healing ability, high tensile strength, and ultra-stretchability.

The lubrication mechanism of human joints and artificial joints is one of the fastest developing aspects in the bio-tribology field. The paper "Biodegradable lubricating mesoporous silica nanoparticles for osteoarthritis therapy" introduces the preparation of biodegradable mesoporous silica nanoparticles (bMSNs) which could overcome the potential toxic effect caused by the bioaccumulation of MSNs. In the paper titled "Investigation of role of cartilage surface polymer brush border in lubrication of biological joints", the authors establish a multi-scale cartilage contact model to study the possible role of the polymer brush boundary (PBB) in cartilage lubrication. Model results suggest that $\mathrm{PBB}$ can significantly enhance the lubrication properties of the cartilage by increasing the gap fluid load and reducing the tissue vertical deformation. The paper "Regulation mechanism of biomolecule interaction behaviors on the superlubricity of hydrophilic polymer coatings" analyzes the effect of biomolecules on the friction behaviors of hydrophilic polymer coatings (poly(vinylphosphonic acid), PVPA). The results indicate protein molecules could form an adsorption film to replace the PVPA surface, in contrast, polysaccharides have zero adsorption on the surface of PVPA, which does not affect the friction properties between the interface.

The paper titled "A microfluidic bleeding model to investigate the effects of blood flow shear on microvascular hemostasis" develops a microfluidic bleeding model and investigate the effect of blood flow shear on microvascular hemostasis. The results indicate the blood flow shear rate could affect the platelet aggregation, which in turn affects the growth and the morphology of the hemostatic thrombus, finally regulating the process of hemostasis.

The paper titled "Robust low friction performance of graphene sheets embedded carbon films coated orthodontic stainless steel archwires" focuses on the friction force between the archwire and bracket used for the orthodontic treatment. The results indicate that in the artificial saliva environment, the carbon film is beneficial for the achievement of a low friction coefficient. In the paper titled "Influence of two polyphenols on the structure and lubrication of salivary pellicle: An in vitro study on astringency mechanism", the authors study the effects of polyphenols on the structure and lubrication of salivary pellicle. Their test results show the structure and physical properties of salivary pellicle change after encountering polyphenolic molecules, which impair its lubrication performance. With the extensive application of $3 \mathrm{D}$ printing technology in the field of clothing and wearable devices, the friction between 3D printing materials and human skin has attracted attention. In the paper titled "Tribological interactions of 3D printed polyurethane and polyamide with water-responsive skin model", the authors prepare TPA/TPU-sodium polyacrylate composites by heat pressing, which reduces the COF in wet conditions. It provides a possible solution to solving the high COF between the 3D printing materials and human skin under wet condition.

Open Access This article is licensed under a Creative Commons Attribution 4.0 International License, which permits use, sharing, adaptation, distribution and reproduction in any medium or format, as long as you give appropriate credit to the original author(s) and the source, provide a link to the Creative Commons licence, and indicate if changes were made.

The images or other third party material in this article are included in the article's Creative Commons licence, unless indicated otherwise in a credit line to the material. If material is not included in the article's Creative Commons licence and your intended use is not permitted by statutory regulation or exceeds the permitted use, you will need to obtain permission directly from the copyright holder.

To view a copy of this licence, visit http://creativecommons.org/licenses/by/4.0/. 